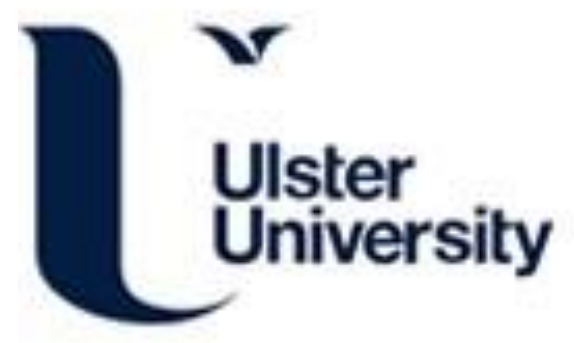

\title{
Exploiting the signatures of nanoplasmon-exciton coupling on proton sensitive insulator-semiconductor devices for drug discovery applications
}

Bhalla, N., \& Estrela, P. (2018). Exploiting the signatures of nanoplasmon-exciton coupling on proton sensitive insulator-semiconductor devices for drug discovery applications. Nanoscale, 10(28), 13320-13328. https://doi.org/10.1039/c8nr04540b

Link to publication record in Ulster University Research Portal

\section{Published in:}

Nanoscale

Publication Status:

Published (in print/issue): 28/07/2018

DOI:

10.1039/c8nr04540b

\section{Document Version}

Author Accepted version

\section{General rights}

Copyright for the publications made accessible via Ulster University's Research Portal is retained by the author(s) and / or other copyright owners and it is a condition of accessing these publications that users recognise and abide by the legal requirements associated with these rights.

\section{Take down policy}

The Research Portal is Ulster University's institutional repository that provides access to Ulster's research outputs. Every effort has been made to ensure that content in the Research Portal does not infringe any person's rights, or applicable UK laws. If you discover content in the Research Portal that you believe breaches copyright or violates any law, please contact pure-support@ulster.ac.uk. 


\section{Journal Name}

\section{COMMUNICATION}

Received 00th January 20xx Accepted 00th January 20xx

DOI: $10.1039 / \times 0 x \times 00000 x$

\section{Exploiting the signatures of nanoplasmon-exciton coupling on proton sensitive insulator-semiconductor devices for drug discovery applications}

\author{
Nikhil Bhalla* ${ }^{\dagger}$ and Pedro Estrela*
}

www.rsc.org/

Multimodal sensing methods have a great promise in biosensing applications as they can measure independently several properties that characterise the biomolecular interaction to be detected as well as providing inherent on-chip validation of the sensing signals. This work describes the mechanisms of a concept of insulator-semiconductor field-effect devices coupled with nanoplasmonic sensing as a promising technology, which can be used for a wide range of analytical sensing applications. The developed method involves coupling of the localized surface plasmons (LSPs) within gold nanoparticles (AuNPs) and excitons within $\mathrm{pH}$ sensitive silicon nitride $\left(\mathrm{Si}_{3} \mathrm{~N}_{4}\right)$ nanofilms for screening inhibitors of kinase, which constitute an important class of chemotherapy drugs. In parallel to this optical sensing, the $\mathrm{pH}$ sensitivity of silicon nitride is used to detect the release of protons associated with kinase activity. By changing the insulator and AuNPs characteristics, this work demonstrates the nanoplasmonicexciton effects taking place, enabling the developed platform to be used for screening kinase inhibitors and as a dual mode electrooptical biosensor for routine bio/chemical sensing applications.

Recent developments in the design and synthesis of plasmonic nanoparticles are based on the discovery of new fundamentals in plasmonic and novel sensing applications of nanomaterials. For instance, recently bimetallic nanoparticles were proposed to solve fundamental material limits associated with oxidation of nanoplasmonic silver ${ }^{1}$. Some advanced applications of such bimetallic nanoparticles include in situ monitoring of catalytic reactions by nanoplasmonic materials ${ }^{2}$. In addition, plasmonic nanoparticles have also been integrated with other nanomaterials such as ferromagnetic oxides to develop composites, which results in simultaneous magnetic

\footnotetext{
${ }^{*}$ Centre for Biosensors, Bioelectronics and Biodevices (C3Bio) and Department of Electronic \& Electrical Engineering, University of Bath BA2 7AY United Kingdom, Email: nikhil.bhalla@bath.edu, P.Estrela@bath.ac.uk

+ Current Address: Micro/Bio/Nanofluidics Unit, Okinawa Institute of Science and Technology Graduate University 1919-1 Tancha, Onna, Kunigami District, Okinawa Prefecture 904-0412 Japan Email: nikhil.bhalla@oist.jp

Electronic Supplementary Information (ESI) available:
}

activity and optical response ${ }^{3}$. Here, the optical property of the whole system could be modulated by application of an external magnetic field leading to highly sensitive nanoparticle-based biosensors. Similarly, the assembly of hybrid superstructures ${ }^{4}$ comprised of biologically active molecules that bind nanoparticles (NPs) to an insulator-semiconductor (IS) structure presents an exploratory biosensing platform with several unique optical and electrochemical properties. While IS primarily enables $\mathrm{pH}^{5,6}$ and charge measurements, the NPs and insulator or their combinatorial interactions give rise to complex optical sensing phenomena ${ }^{7}$. For instance, localized surface plasmon resonance (LSPR) ${ }^{8}$, surface enhanced Raman spectroscopy $(\mathrm{SERS})^{9}$, plasmon-exciton interactions ${ }^{10}$, phononplasmon combination ${ }^{11}$, magneto-plasmonic coupling ${ }^{12}$ and luminescence enhancement ${ }^{13}$ have been observed in these NPbased hybrid superstructures. Therefore, NP-IS hybrids can be considered as an enabling technology for dual-mode optoelectronic biosensors that can take advantage of both fieldeffect devices and nonlinear optical sensing properties of the system. In this dual mode detection, both sensing mechanisms are independent and complementary to each other (see figure 1). This makes the sensor robust for biosensing applications, where one technique validates the other ${ }^{14}$. We here focus on the use of such platforms for generic biosensing applications by demonstrating a kinase assay for drug discovery applications ${ }^{15-}$ 18. More importantly, we show how coupling of excitons and plasmons vary by changing the sizes of gold nanoparticles (AuNPs), while characterizing the $\mathrm{pH}$ sensing properties of the surface.

When $\mathrm{Si}_{3} \mathrm{~N}_{4}$-based IS structures without AuNPs are exposed to white light between $400-800 \mathrm{~nm}$, excitons are generated due to the radiative and non-radiative recombination of electronhole pairs in the $\mathrm{Si}_{3} \mathrm{~N}_{4}$ top layer ${ }^{19}$. This happens as a result of optical pumping of the oxide-insulator interface $\left(\mathrm{SiO}_{2}-\mathrm{Si}_{3} \mathrm{~N}_{4}\right)$ and insulator trapped charges in $\mathrm{Si}_{3} \mathrm{~N}_{4}$ triggered by multiple reflections at the interface of each layer on the wafer ${ }^{20}$. The presence of these excitons, well established in literature ${ }^{19,21}$, is validated by capacitance - voltage $(C-V)$ profiling of IS junctions 
as shown in figure $2 a . C-V$ measurements were carried out with a $1 \mathrm{kHz}$ frequency, $10 \mathrm{mV} A C$ signal superimposed on a $D C$ sweep from -1.0 to $3.0 \mathrm{~V}$ both in the presence and absence of light. Without light, there is a formation of distinct inversion, depletion and accumulation regions in the $C-V$ characteristics of the electrolyte-insulator-semiconductor (EIS) structure. Inversion capacitance is formed as the minority carriers in the inversion region are not able to respond to the fast changes in the $A C$ voltage. However, when light is shinned on the surface of the silicon nitride there is a formation of excitons, i.e. recombination of electrons and holes that takes place. This recombination of the charge carriers (electrons and holes) is reflected with increase in inversion capacitance in the presence of light (like a low-frequency Metal-Oxide-Semiconductor capacitor response).

In addition, a change in the threshold potential of the IS device (from $0.97 \mathrm{~V}$ without light to $0.92 \mathrm{~V}$ upon exposure to light) is also observed suggesting that there is an optically induced pumping of charge carriers at oxide-insulator interface. The resulting extinction of this phenomenon gives rise to an absorption peak in $\mathrm{Si}_{3} \mathrm{~N}_{4}{ }^{22}$ as shown in figure $2 \mathrm{~b}$. The fact that this particular peak reflects excitons is validated with another simple experiment in which the IS substrate is cooled down by dipping it in liquid nitrogen for 2 minutes and then spontaneously measuring the optical response (figure $2 \mathrm{c}$ ).

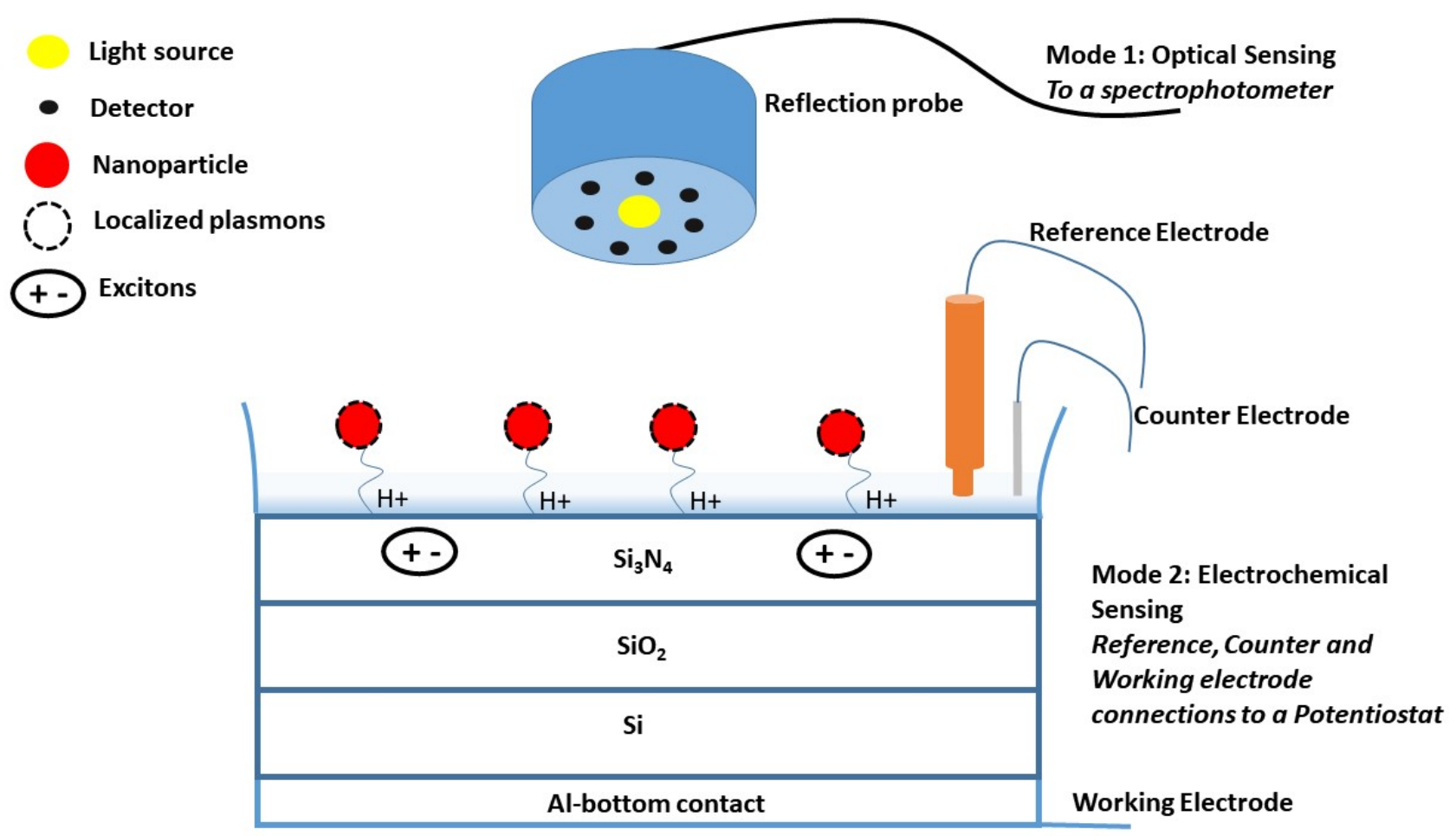

Figure. 1. Scheme for the dual-mode biosensor: Mode 1 is the optical sensing where the reflection probe shows integrated light source and detector to measure light absorbance from the surface. Mode 2 shows an electrochemical sensing setup with a conventional 3-electrode setup to measure the capacitance of the electrolyte-insulator-semiconductor structure.

The recombination rates of electrons and holes change with changes in temperature of the IS structures and therefore a change in optical extinction spectrum (leading to change in absorbance) of the substrate is expected. Upon cooling, a 20\% increase is observed in the full width half maxima (FWHM) of the absorbance spectra for the IS structure, from $82 \mathrm{~nm}$ to 98 $\mathrm{nm}$, indicating a decrease in recombination of charge carriers. Once the IS substrate returns back to room temperature $\left(25^{\circ} \mathrm{C}\right)$ in 4-5 minutes, the absorbance peak also returns back to its original position, indicating that the peak in figure $2 b$ contains signatures of excitons.

We observe that this exciton-related absorbance peak of the $\mathrm{Si}_{3} \mathrm{~N}_{4}$ layer is enhanced by immobilization of AuNPs on the top of $\mathrm{Si}_{3} \mathrm{~N}_{4}$, which is explained by the coupling between excitons and AuNPs localized surface plasmons (LSPs) as observed in the literature for silver nanostructures ${ }^{23}$ (see figure $2 \mathrm{~d}$ ). Briefly, by adding nanoparticles of 5,10 and $20 \mathrm{~nm}$, redshifts in the properties of $\mathrm{Si}_{3} \mathrm{~N}_{4}$ are observed. This coupling is considered as a two-step process where excitons in the $\mathrm{Si}_{3} \mathrm{~N}_{4}$ matrix first transfer their energies to LSP modes, which then outcouple to propagation modes, enhancing the optical absorbance signal from the excitons in the $\mathrm{Si}_{3} \mathrm{~N}_{4}$ matrix ${ }^{19,23}$. We also observe that by simply varying the thickness and composition of the insulator, the strength of this exciton-plasmon coupling can be modified, leading to versatile characteristics of the proposed biosensor platform. To validate this hypothesis, we made 3 more IS structures with completely different morphologies from each other as shown in table 1. Microscopy and spectroscopy 
scans to characterise the structural and material properties of the wafers are included in the supplementary information. All four wafers appear to have different colour where the thickness of $\mathrm{Si}_{3} \mathrm{~N}_{4}$ layer was varied between 100 to $400 \mathrm{~nm}$ while varying the stoichiometric ratio of nitrogen and oxygen. Thicknesses of $\mathrm{Si}_{3} \mathrm{~N}_{4}$ layers in all wafers were controlled during the PECVD process and the cross-section of the wafer was measured after the completion of deposition using SEM. EDX, XPS and FTIR spectroscopy studies revealed slight variations in the composition of the silicon nitride layer, see details in supplementary information, figure $3 a \& 3 b$ and table 1.

These property variations were attained by different ratios of nitrogen and oxygen in the silicon nitride layer and therefore a mixed presence of $\mathrm{Si}-\mathrm{H}, \mathrm{Si}-\mathrm{O}, \mathrm{N}-\mathrm{H}$ and $\mathrm{Si}-\mathrm{N}$ bonds is observed in the different samples, see figure $3 a$ for FTIR and $3 b$ for XPS characterization and table 1 for atomic percentage of elemental composition of the samples. Figure 4 a shows the optical response of all 4 IS structures. There are two resonance peaks observed in the 3 wafers with a $\mathrm{SiO}_{2}$ thickness of 35-50 nm and $\mathrm{Si}_{3} \mathrm{~N}_{4}$ between 100-200 nm (wafers \#2, \#3 and \#4). The first peak is between $400-450 \mathrm{~nm}$, while the second one is around $820-$ $830 \mathrm{~nm}$. With increase of the $\mathrm{Si}_{3} \mathrm{~N}_{4}$ thickness to $400 \mathrm{~nm}$ and decrease in the thickness of $\mathrm{SiO}_{2}$ to $10 \mathrm{~nm}$, there is only one resonance peak of $\mathrm{Si}_{3} \mathrm{~N}_{4}$ at $750 \mathrm{~nm}$ (wafer \#1). It should be noted that no correlation between characteristic peaks of wafers \#1, $\# 2$, \#3 and \#4 and the structural composition of $\mathrm{Si}_{3} \mathrm{~N}_{4}$ can be extracted from the experiments in this study and that more systematic studies are required in the future to deduce thickness dependency of optical absorbance in $\mathrm{Si}_{3} \mathrm{~N}_{4}$. In addition, an increased amount of disorder in the grains and large lattice strain in $\mathrm{Si}_{3} \mathrm{~N}_{4}$ is seen when its thickness is increased, relative to other wafers as analysed from XRD scans.

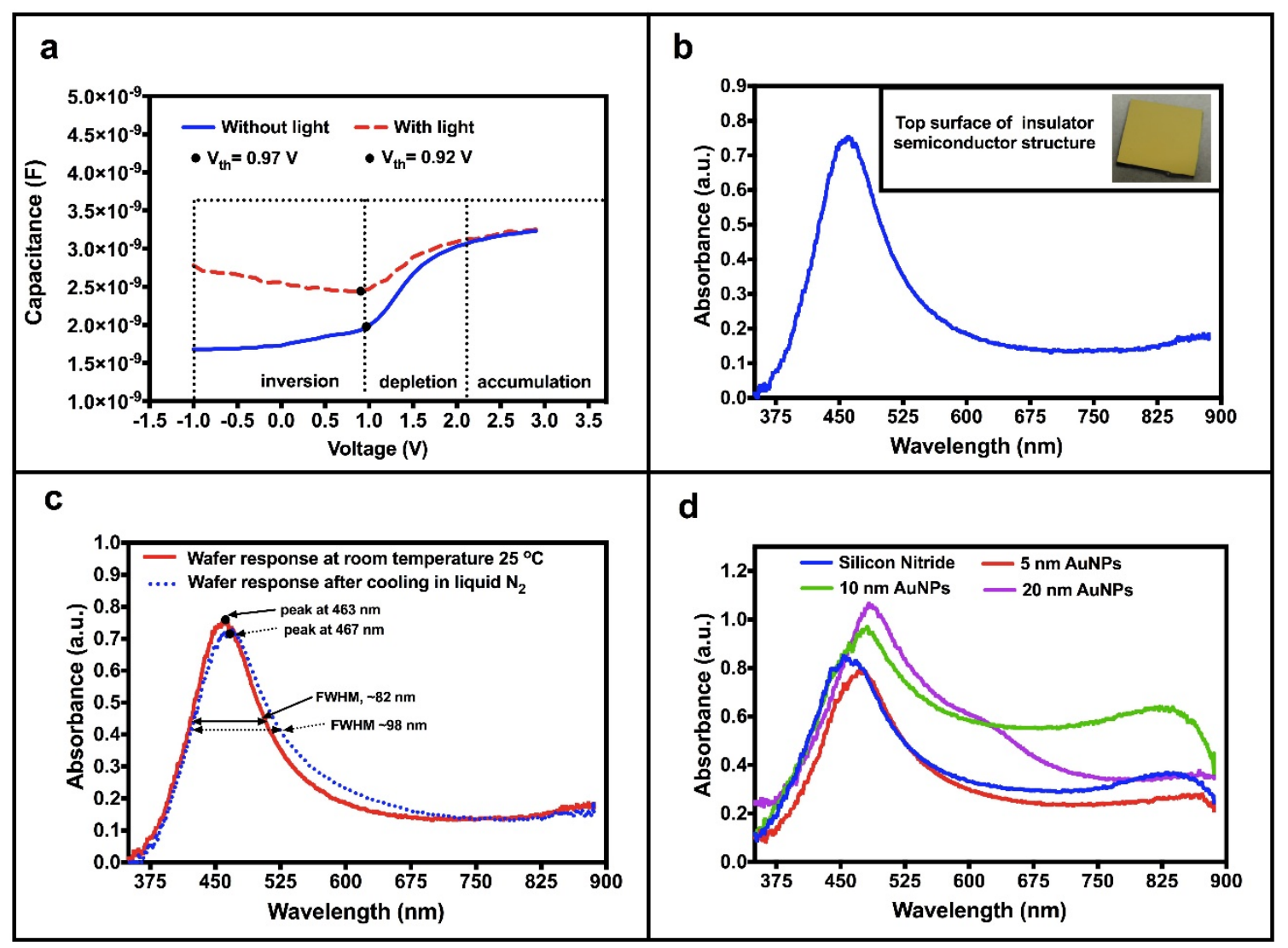

Figure. 2. a) Capacitance Voltage characteristics of insulator semiconductor (IS) wafer in the presence and absence of lights, b) Optical absorbance of the IS wafer in reflection mode, c) effect on optical absorbance of IS wafer upon instantaneous cooling of wafer in liquid nitrogen, d) Absorbance characterization of IS wafer without AuNPs attached to $\mathrm{Si}_{3} \mathrm{~N}_{4}$ and with 5,10 and $20 \mathrm{~nm}$ of AuNPs attached on $\mathrm{Si}_{3} \mathrm{~N}_{4}$ layer of IS wafer.

Evidently, it is the different structural properties and composition of $\mathrm{Si}_{3} \mathrm{~N}_{4}$ (see all wafer properties in table 1 ) that yields absorbance peaks at different wavelengths, as shown in figure 4a. Moreover, compositional dependence of absorbance peaks due to excitonic resonances in $\mathrm{Si}_{3} \mathrm{~N}_{4}$ has also been reported in literature by Kistner et $a l^{22}$. In their work they reported that the resonances in $\mathrm{Si}_{3} \mathrm{~N}_{4}$ depend strongly on the silane/ammonia ratio. The resonances observed in 3 of our wafer samples between 400-450 nm would correspond to $\mathrm{R} \sim 1 \%$, where $\mathrm{R}=\left[\mathrm{SiH}_{4}\right] /\left[\mathrm{NH}_{3}\right]$, silane/ammonia ratio. Similarly, the peak observed at $750 \mathrm{~nm}$ in wafer \#1 corresponds to $\mathrm{R} \sim 20 \%$. Kistner et al. also reported that the peaks change drastically for different $R$ values, which is consistent with our results. 
Now when the AuNPs are chemically attached to the surface there is an enhancement of the absorption peak. Higher absorbance, widened FWHM and a redshift in the wavelength of the are main characteristics of changes observed in the peak, see figure $4 \mathrm{~b}-\mathrm{e}$. The redshift can be ascribed to the Purcell effect $^{24}$, where the radiative recombination energies of the electron-hole pairs in $\mathrm{Si}_{3} \mathrm{~N}_{4}$ are enhanced by the strong electromagnetic (EM) fields introduced by high density mode localized surface plasmons in AuNPs. Increased redshift is also observed in the peak with increase in the size of the nanoparticles (figure $4 \mathrm{~b}-\mathrm{e}$ ). This is attributed to the following points: 1 ) the larger size of the AuNPs decreases the distance between NPs and $\mathrm{Si}_{3} \mathrm{~N}_{4}$, indicating strong interaction between them. This is valid if we assume that the centre of nanoparticles attaches to the same location on the protein. The closer the source of excitons is to the source of plasmons, the stronger is the coupling of their evanescent fields.; 2 ) the density of LSP modes increases with the size of AuNPs, which confines stronger EM fields on the $\mathrm{Si}_{3} \mathrm{~N}_{4}$ surface; and 3 ) there is an enhancement in the Purcell factor with increase in size of nanoparticles, which enhances the exciton-plasmonic coupling. Interestingly, the amount of redshifts upon addition of AuNPs was different for all wafers which is attributed to the different compositions of $\mathrm{Si}_{3} \mathrm{~N}_{4}$. With increase in the thickness of $\mathrm{Si}_{3} \mathrm{~N}_{4}$, the redshifts of exciton-plasmon coupled system (i.e. after addition of AuNPs) also increase as seen for wafer \#1 in figure $4 \mathrm{~b}$ and figure $4 \mathrm{e}$. This is associated with the fact that relatively higher number of recombination would occur in a thick $\mathrm{Si}_{3} \mathrm{~N}_{4}$ layer due to rather higher proportions of bulk $\mathrm{Si}_{3} \mathrm{~N}_{4}$.

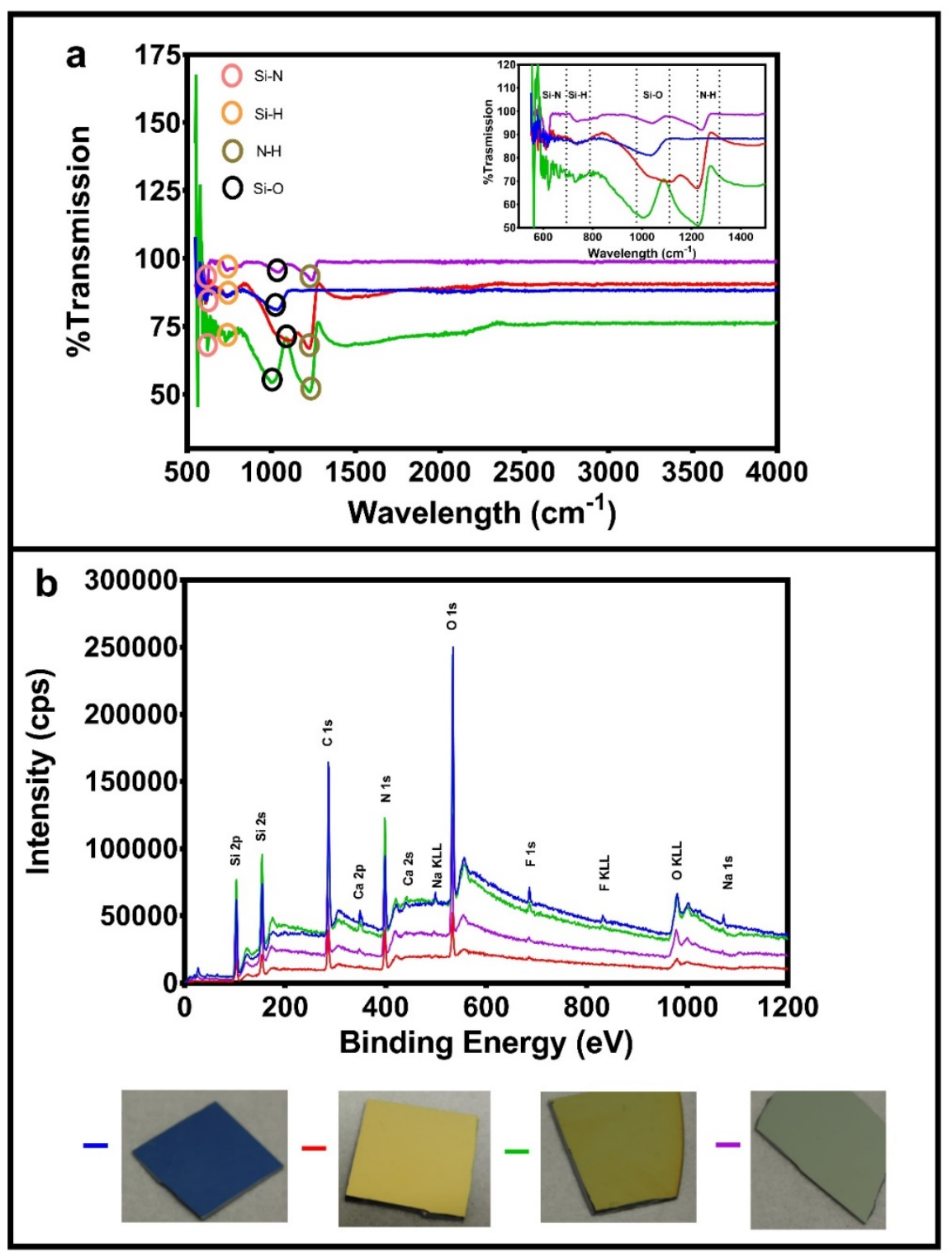

Figure. 3. a) FTIR and b) XPS characterization of all four wafers fabricated to study exciton plasmon coupling. Note: the legends shown underneath the graph $3 b$ apply for both graphs $3 a \& 3 b$ as same colour code is used to represent the data of respective wafer. 


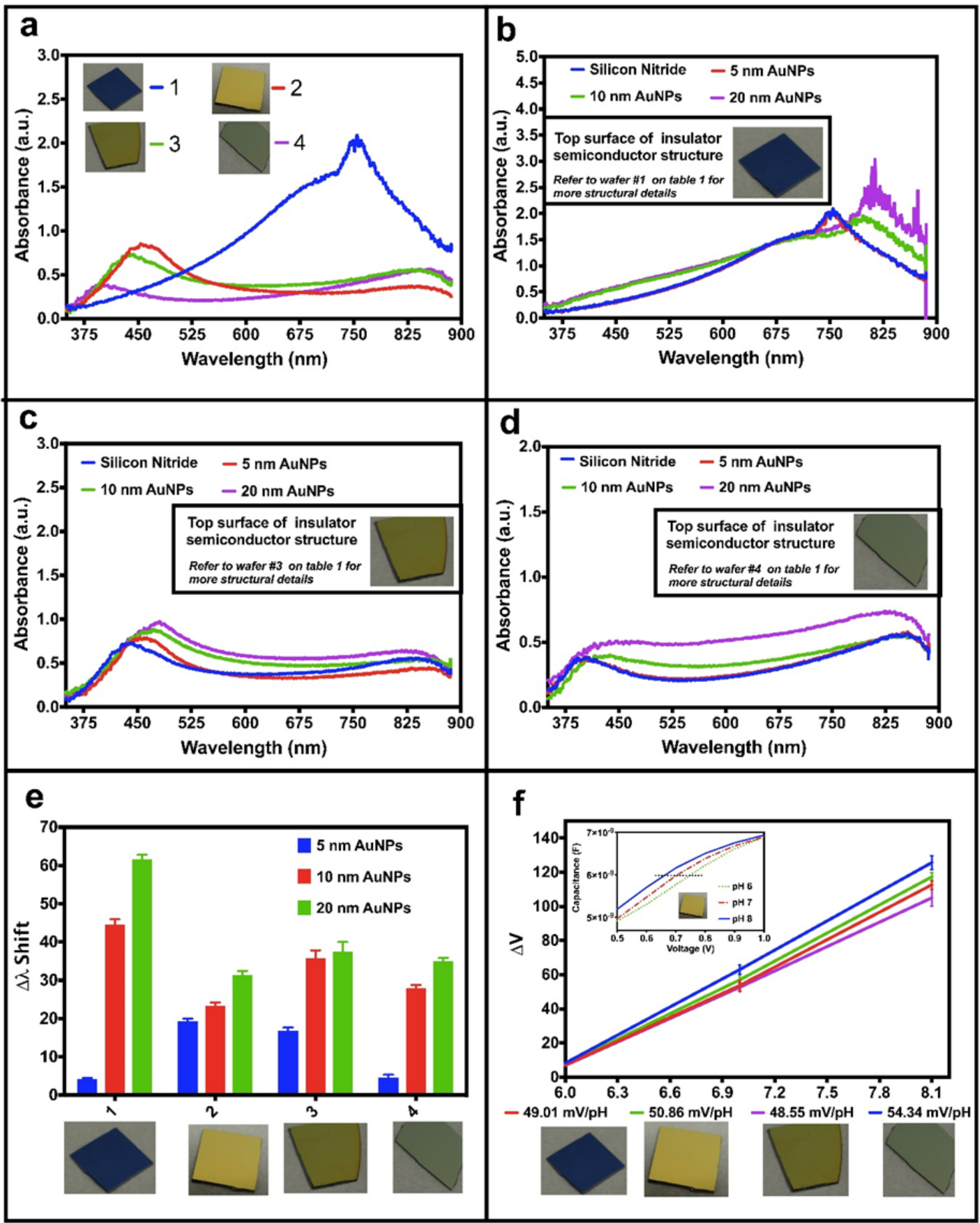

Figure. 4. a) shows optical absorbance characteristics of all four wafers fabricated to study exciton plasmon coupling. b-d) shows the effect on the absorbance characteristics of IS wafer upon attachment of AuNPs of various sizes. e) shows a histogram with statistical data comparing 4 wafers used in the study and f) shows $\mathrm{pH}$ response of 4 IS structures.

However, we observe that this wavelength change response to the size of the AuNPs is not comparable from one wafer to the other. For instance, in wafers \#1 and \#4, the excitonplasmon coupling is not found to be effective as in wafers \#2 and \#3, when the nanoparticle size is small $(5 \mathrm{~nm})$, see figure $4 b, d$ and e. This is an anomalous behaviour of the wafers, which is attributed to the differences in the composition of the
4 wafer suppliers. This behaviour is anomalous because ideally the wafers with higher nitrogen content (which is not suggested by composition of \#1 and \#4 from XPS and FTIR) should have weaker exciton-plasmon. This is due the well-known fact that the ionization energy of nitrogen atom is higher than that of oxygen atom leading to a phenomenon where the nitrogen 
requires more energy relative to oxygen for discharging an electron from its valence shell.

While the composition and structural properties of the silicon nitride largely affect the optical response of the substrates, it is observed that the $\mathrm{pH}$ sensitivity of the substrates doesn't change drastically (figure $4 \mathrm{f}$ ). A difference of $10.5 \%$ between the most sensitive and the least $\mathrm{pH}$ sensitive $\mathrm{Si}_{3} \mathrm{~N}_{4}$ is observed in comparison to $100 \%$ changes in the optical enhancements between the samples with the best and the worst wavelength redshifts. This is primarily because the composition of $\mathrm{Si}_{3} \mathrm{~N}_{4}$ does not significantly affect the hydration sites (dangling hydroxyl-OH bonds) on the nitride surface.
This physical characterization of 4 substrates suggests that while these IS structures can be used to measure optical response and $\mathrm{pH}$ changes associated with some chemical reaction happening on the surface, it is important to optimize the fabrication parameters and/or choose nanoparticle size which provides maximum enhancement in the exciton-plasmon coupling. Therefore, from the above material characterization we chose wafer \#2 for carrying out kinase assays and demonstrate how a simple dual mode biosensing can be achieved to discover inhibitors of kinases which are essentially the main drugs used in chemotherapy treatment for a wide range of cancers.

Table. 1. Characteristics of 4 IS wafers used in this study

\begin{tabular}{|c|c|c|c|c|c|c|c|c|c|c|c|c|}
\hline \multirow[t]{2}{*}{ Wafer } & \multirow{2}{*}{$\begin{array}{c}\text { Average } \\
\text { thickness of } \\
\mathrm{Si}_{3} \mathrm{~N}_{4} \text { and } \mathrm{SiO}_{2} \\
\text { on } \mathrm{Si} \text { wafer } \\
(\mathrm{nm})\end{array}$} & \multicolumn{7}{|c|}{$\begin{array}{l}\text { Elemental composition on the top } \\
\qquad \begin{array}{l}\mathrm{Si}_{3} \mathrm{~N}_{4} \text { layer } \\
(\% \text { atoms })\end{array}\end{array}$} & \multirow{2}{*}{$\begin{array}{l}\text { Approximate } \\
\mathrm{N}: \mathrm{O} \text { ratio on the } \\
\text { top } \mathrm{Si}_{3} \mathrm{~N}_{4} \text { layer } \\
\text { (based on XPS) }\end{array}$} & \multirow[t]{2}{*}{ FTIR peaks } & \multirow{2}{*}{$\begin{array}{l}\text { Lattice } \\
\text { spacing as } \\
\text { seen from } \\
\text { XRD (Å) }\end{array}$} & \multirow{2}{*}{$\begin{array}{l}\text { Intensity } \\
\text { of [321] } \\
\text { peaks in } \\
\text { XRD (a.u.) }\end{array}$} \\
\hline & & C & 0 & $\mathbf{N}$ & Si & $\mathbf{F}$ & $\mathrm{Ca}$ & $\mathrm{Na}$ & & & & \\
\hline & $\begin{array}{c}\mathrm{Si}_{3} \mathrm{~N}_{4}: 400-410 \\
\mathrm{SiO}_{2}: 5-10\end{array}$ & 45 & 23 & 11 & 18 & 1 & 1 & 1 & $1: 2$ & $\begin{array}{l}\text { Si-H, Si-O, Si- } \\
N\end{array}$ & $d_{321}=1.36$ & 35 \\
\hline & $\begin{array}{c}\mathrm{Si}_{3} \mathrm{~N}_{4}: 102-113 \\
\mathrm{SiO}_{2}: 45-50\end{array}$ & 38 & 18 & 24 & 18 & $<1$ & $<1$ & $<1$ & $4: 3$ & $\begin{array}{l}\text { Si-H, Si-O, N- } \\
\mathrm{H}, \mathrm{Si}-\mathrm{N}\end{array}$ & $\begin{array}{l}d_{321}=1.35 \\
d_{101}=2.70\end{array}$ & 160000 \\
\hline 3 & $\begin{array}{c}\mathrm{Si}_{3} \mathrm{~N}_{4}: 160-170 \\
\mathrm{SiO}_{2}: 45-50\end{array}$ & 33 & 25 & 17 & 24 & $<1$ & $<1$ & $<1$ & $3: 4$ & $\begin{array}{l}\text { Si-H, Si-O, N- } \\
\mathrm{H}, \mathrm{Si}-\mathrm{N}\end{array}$ & $\begin{array}{l}d_{321}=1.35 \\
d_{101}=2.71\end{array}$ & 53000 \\
\hline & $\begin{array}{c}\mathrm{Si}_{3} \mathrm{~N}_{4}: 100-115 \\
\mathrm{SiO}_{2}: 35-40\end{array}$ & 30 & 24 & 22 & 23 & $<1$ & $<1$ & $<1$ & 1:1 & $\begin{array}{l}\mathrm{Si}-\mathrm{H}, \mathrm{Si}-\mathrm{O}, \mathrm{N}- \\
\mathrm{H}, \mathrm{Si}-\mathrm{N}\end{array}$ & $\begin{array}{l}d_{321}=1.35 \\
d_{101}=2.70\end{array}$ & 185000 \\
\hline
\end{tabular}

Kinase inhibitors constitutes a class of chemotherapy medications that inhibit function of kinases which are primarily involved in phosphorylation of proteins. Phosphorylation is one of the most common post translational modification of portions where the kinases mediate the release of phosphate group from phosphate donating molecules such as ATP. The reaction involves addition of phosphate group to mainly 4 amino acids (serine, threonine, tyrosine and histidine) if present within a given protein. While phosphorylation is important to maintain necessary cell function, for instance in glycolysis, it is often reported as a cause or a consequence of many fatal diseases such as cancer ${ }^{25}$, dementia ${ }^{26}$ and Alzheimer's disease ${ }^{27}$. Therefore, kinase assays represent promising tools to screen chemotherapy drug targets and the development of new/improved screening methodologies is the evergreen need of pharmaceutical industry.

To detect phosphorylation of proteins one of the three sensing strategies can be applied to confirm the addition of phosphate group to amino acids with in protein/peptide: 1) by measuring the change in the charge of the protein/peptide after addition of phosphate group, 2) by detecting the release of protons associated with phosphorylation of proteins (during conversion of ATP to ADP) and 3) by modification at the $\gamma$ phosphoryl group of ATP with a group that generates either an optical or electrical response. For example, in this work we modify the terminal phosphoryl group of ATP with a sulphide bond (ATP-S), which allows attachment of AuNPs to the phosphorylated proteins. Since the phosphorylated proteins consist of phosphoryl group linked with a sulphide, we use the term thio-phosphorylation of proteins to define this reaction. During thio-phosphorylation there is a release of proton which is detected by $\mathrm{pH}$ sensitive silicon nitride surfaces and in addition AuNPs can be added to the system, attaching specifically to the thio-phosphorylated proteins, and hence enabling an exciton-plasmonic coupled optical signal from silicon nitride and AuNPs combination, only present when phosphorylation occurred.

Figure 5 a shows the charge change on the surface of $\mathrm{pH}$ sensitive insulator-semiconductor structure response upon thio-phosphorylation of in-house synthesised peptides. Two peptides, described in the methods section, one with cysteine and another without cysteine amino acids were obtained from Department of Pharmacy and Pharmacology, University of Bath. Peptides were immobilised on the surface of silicon nitride with (3-glycidyloxypropyl) trimethoxysilane (GOPTS) linker molecules and subsequently the peptides were exposed to a mixture a solution consisting of kinase, ATP and with/without kinase inhibitors and activators. The difference in the 2 
peptides, peptide-1 (NH2-CRRRKGSFRRKK-COOH) and peptide2 (NH2-RRRKGSFRRKK-COOH) is the presence of a cysteine amino acid in one of the peptides.

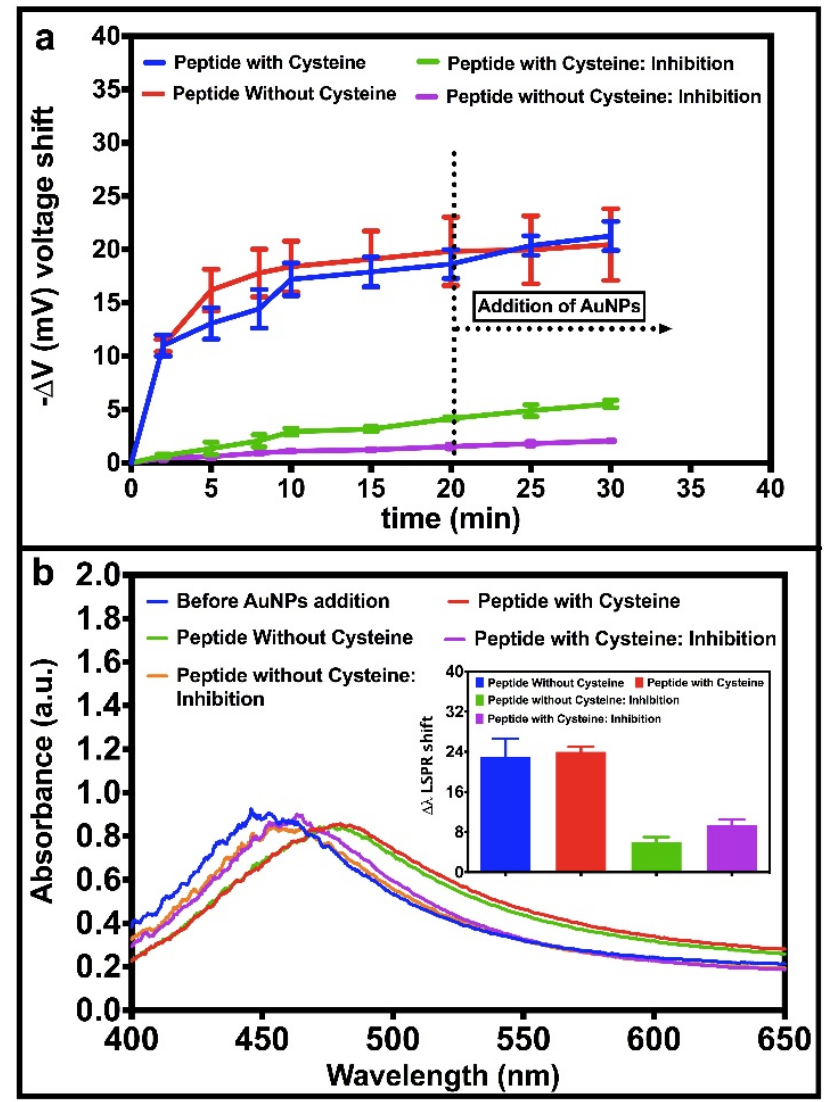

Figure. 5. a) Electrochemical sensing mode for the detection of thio-phosphorylation of peptides. b) Optical sensing mode based on LSPR for the detection of thio-phosphorylation events. The inset shows statistics for experiment replicates $(n \geq 3)$

A large number of proteins have a cysteine amino acid as one of their components. AuNPs have a high affinity to the sulphur group present in the cysteine amino acid. Therefore, it was necessary to study the interaction of AuNPs with the cysteine amino acid to understand how AuNPs could attach to a protein even without thio-phosphorylation, which would give false positive results to our sensor. As shown in figure $5 \mathrm{a}, \mathrm{a}$ mean value of $20 \mathrm{mV}$ was observed after thio-phosphorylation of both the peptides.

In the presence of inhibitor, shifts $<5 \mathrm{mV}$ in the IS signal were observed. These shifts are attributed to the $\mathrm{pH}$ changes associated with the thio-phosphorylation of peptides. The signal after the dotted line shows the changes after addition of AuNPs. Figure $5 b$ shows results from optical sensing mode of the sensor. Due to presence of LSPR on AuNPs, redshifts of $\sim 25$ $\mathrm{nm}$ were observed upon attachment of AuNPs with the thiophosphorylated peptides (Figure 5-b), which consists of excitonplasmonic coupled signal as discussed above. This signal was comparatively more reproducible from the peptides with cysteine than that from the peptide without cysteine. This is due to the non-specific attachment of the AuNPs on the cysteinecontaining peptides. However, it is evident from Figure $5 b$, inset that the non-specific attachment of the AuNPs do not contribute to significant signal changes in the optical signal and the thio-phosphorylation reaction can easily be distinguished from the inhibition reaction. The results suggest that the presence of a non-specific interaction may lead to periodicity in the distribution of AuNPs which contributes to less variation in the optical response from sample to sample.

Moreover, two modes of the sensor (electrochemical: electrolyte-insulator-semiconductor capacitance and optical: LSPR) have distinguishable inhibition and phosphorylation reactions, suggesting that the platform can be used for screening novel inhibitors.

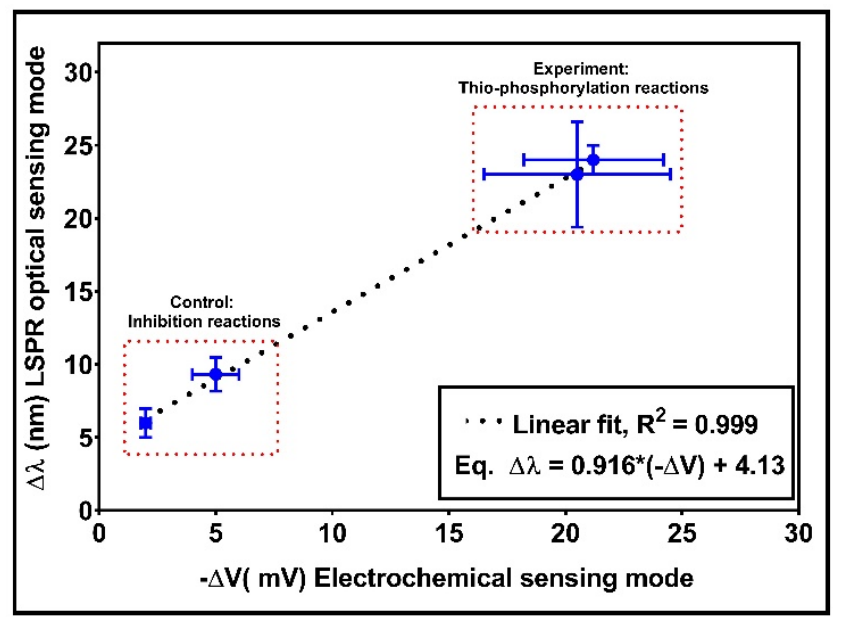

Figure. 6. Correlation between electrochemical and optical sensing modes. Please note that this fitting data is valid only for the kinase assay demonstrated in this work. The equation does not provide an absolute relation between LSPR and EIS (electrolyte insulator semiconductor) sensing system

The electrochemical mode of the platform also allows the user to follow the kinetics of phosphorylation as seen from the results in figure $5 \mathrm{a}$. Following kinetics of inhibition or phosphorylation reaction will provide information on efficacy of the drug under test which is important to understand how a particular drug functions.

In addition, we also find a relationship between the signals of the two modes. In figure 6 , we plot the output of both electrochemical signal and optical signals for all measurements done for both thio-phosphorylation and inhibition experiment. We observe that the data can be fitted with a linear relationship with a regression coefficient, R-squared value, of 0.999 . This also implies that both LSPR and electrochemical modes respond to the change in sensor surface linearly in a complementary manner, thus increasing reliability of the measurement

This dual mode detection method that we propose can easily be extended to multiplexed approaches for high throughput analyses of protein kinase activity. As a result, the screening of protein kinase inhibitors becomes more rapid, sensitive, robust and cost-effective representing advancement in the development of concepts and devices for discovering 
potential chemotherapy drugs. The work here presented further demonstrates the mechanisms involved in the detection and the importance of careful device fabrication for optimal sensing.

\section{Methods}

To conduct experiments aqueous solutions were made with doubly de-ionised water, $18.2 \mathrm{M} \Omega \mathrm{cm}$, with a Pyrogard filter (Millipore, USA). $\mathrm{Si}_{3} \mathrm{~N}_{4} / \mathrm{SiO}_{2}$ layers were deposited on the silicon wafers using a plasma enhanced chemical wafer deposition (PECVD) process. The process for cleaning and preparation of wafers for measurements has been reported in our previous work $^{25}$. Silicon nitride layers were modified with $25 \%$ mercaptopropyl trimethoxysilane (MPTES) in absolute ethanol in order to create thiol groups on the surface for direct chemical attachment of AuNPs. All chemicals, AuNPs and other buffer reagents were bought from Sigma Aldrich (UK) and used as received, unless specified. Optical interaction of the AuNPs with the $\mathrm{Si}_{3} \mathrm{~N}_{4}$ was measured using a reflection mode LSPR system built in-house ${ }^{29}$. Briefly, the wafers were exposed to light using an LS-1-LL Tungsten halogen source (Ocean Optics, USA) positioned perpendicularly to their surface using a reflection probe R400-7-UV-VIS (Ocean Optics, USA). The optical signal was collected by the reflection probe USB400-UV-VIS-ES spectrophotometer (Ocean Optics, USA). Capacitance-Voltage measurements for $\mathrm{pH}$ sensing were carried out using a CompactStat potentiostat (Ivium Technologies, Netherlands). Scanning electron microscopy (SEM) was performed on a JEOL SEM6480LV and energy dispersive X-ray analysis (EDX) was carried out using a high sensitivity Oxford INCA X-Act SDD X-ray detector equipped with the SEM. Fourier Transform Infrared Spectroscopy (FTIR) was performed directly over the $\mathrm{Si}_{3} \mathrm{~N}_{4}$ surface using a Perkin Elmer Frontier FTIR instrument in reflection mode with a high resolution MCT (Mercury Cadmium Telluride) detector. X-Ray Diffraction (XRD) data was obtained using a Bruker AXS D8 Advance equipped with a Vante-1 detector using CuK $\alpha$ radiation $(\lambda=1.5318 \AA$ ) .

\section{Kinase assay}

Kinase assays to study protein phosphorylation studies were carried out on peptides. A peptide is a smaller molecule than protein with 50 or fewer amino acids. Two peptides were synthesised to study the kinase activity. Both peptide-1 (NH2CRRRKGSFRRKK-COOH) and peptide-2 (NH2-RRRKGSFRRKK$\mathrm{COOH}$ ) were prepared with one phosphorylation site (serine amino acid). The only difference between peptide- 1 and peptide- 2 was the presence of an extra amino acid, cysteine, in peptide-1. The composition of both peptides was confirmed using mass spectroscopy studies. The purity levels of the prepared peptides were found to be above $85 \%$, which was revealed by high performance liquid chromatography (HPLC).

A. Reagents: All chemicals were of analytical grade and were used as received. The aqueous solutions were made with double de-ionised water, $18.2 \mathrm{M} \Omega \mathrm{cm}$, with a Pyrogard filter (Millipore, USA). Tris base, $\mathrm{MgCl}_{2}, \mathrm{NaCl}$, acetone, $\mathrm{NH}_{4} \mathrm{OH}, \mathrm{HCl}$, $\mathrm{H}_{2} \mathrm{O}_{2}$, 3-glycidoxypropyltrimethoxysilane (GOPTS), PKC- $\alpha$ kinase inhibitor GF 109203X and adenosine 5'-[ $\gamma$-thio] triphosphate (ATP-S) were purchased from Sigma-Aldrich. Dephosphorylated myelin basic protein (MBP), purified from bovine brain using FPLC liquid chromatography, was purchased from Millipore. Protein Kinase C alpha (PKC- $\alpha$ ) human recombinant kinase produced in Sf9, was procured from ProSec-Tray TechnoGene Ltd. PKC lipid activator cocktail was obtained from Millipore. All size ( $5 \mathrm{~nm}, 10 \mathrm{~nm}$ and $20 \mathrm{~nm}$ ) gold nanoparticles (AuNPs) were of research grade and were bought in colloidal form from Diagnostic Consulting Network.

B. Sample preparation and measurement setup: The $\mathrm{Si}-\mathrm{SiO}_{2}-$ $\mathrm{Si}_{3} \mathrm{~N}_{4}$ wafer was cleaned using a standard RCA wafer cleaning protocol. Briefly, the wafer was firstly rinsed with ultra-pure deionised (DI) water and then immersed in 1:1:5 solution of $\mathrm{NH}_{4} \mathrm{OH}: \mathrm{H}_{2} \mathrm{O}_{2}: \mathrm{H}_{2} \mathrm{O}$ at $80^{\circ} \mathrm{C}$ for $10 \mathrm{~min}$ to remove organic contaminants. In the next step, to remove inorganic contaminants, the wafer was socked in 1:1:6 solution of $\mathrm{HCl}: \mathrm{H}_{2} \mathrm{O}_{2}: \mathrm{H}_{2} \mathrm{O}$ at $80{ }^{\circ} \mathrm{C}$ for $10 \mathrm{~min}$; finally the wafer was rinsed with DI. After cleaning the wafer, $100 \mathrm{~nm}$ aluminium was physically deposited on the back of the Si wafer to serve as an ohmic back-contact using an Edwards e-beam evaporator. The wafer was sandwiched between a teflon well with an o-ring and a conductive plate (copper), so that the aluminium coated side of the wafer sits on the lower conductive plate. More details of the teflon well can be referred on the supplementary information of our previous work ${ }^{28}$.

C. Thio-phosphorylation reaction: First, the peptides were immobilised on the surface of silicon nitride with GOPTS linker molecules and subsequently the peptides were exposed to a mixture a solution consisting of kinase, ATP and with/without kinase inhibitors and activators. This reaction was carried out in low ionic strength buffer $(0.2 \mathrm{mM}$ Tris base, $\mathrm{pH} 7.4,6 \mathrm{mM} \mathrm{NaCl}$ and $0.4 \mathrm{mM} \mathrm{MgCl} 2$ ). The whole reaction volume was fixed to $100 \mu \mathrm{l}$ for all replicates and their controls. $1 \mu \mathrm{M}$ ATP-S and 4 units of PKC- $\alpha(40 \mathrm{mU} / \mu \mathrm{l}))$ were subsequently added. To initiate the phosphorylation reaction, PKC lipid activator (1:20 of reaction volume) containing $0.5 \mathrm{mg} / \mathrm{ml}$ phosphatidylserine and $0.05 \mathrm{mg} / \mathrm{ml}$ diacyglycerol in $20 \mathrm{mM}$ MOPS (pH 7.2), $25 \mathrm{mM} \beta$ glycerol phosphate, $1 \mathrm{mM}$ sodium orthovanadate, $1 \mathrm{mM}$ dithiothretiol and $1 \mathrm{mM} \mathrm{CaCl}$, was added. The control experiment was performed with $0.1 \mu \mathrm{M}$ PKC kinase inhibitor (GF 109203X), which were added before adding the kinase activator.

D. Capacitance-Voltage measurements: PKC- $\alpha$ activity was measured by analysing the capacitance-voltage $(C-V)$ characteristics of the $\mathrm{Si}_{3} \mathrm{~N}_{4} / \mathrm{SiO}_{2} / \mathrm{Si}$ EIS structure. $C-V$ measurements were performed using a CompactStat digital potentiostat (Ivium Technologies, The Netherlands). A conventional three-electrode electrochemical setup was employed with an $\mathrm{Ag} / \mathrm{AgCl}$ reference electrode immersed in the electrolyte via a salt bridge used to apply the gate voltage and a Pt counter electrode. During the measurements, the gate voltage $(V \mathrm{~g}$, applied between the reference electrode and the Al back-contact) was varied between -1 and $+3 V$, superimposed 
with a small ac signal of $10 \mathrm{mV}$ at $1 \mathrm{kHz}$. The first measurement for the reaction was taken after adding ATP-S and kinase, i.e. before the start of phosphorylation process. After adding the kinase activator, the activity of the reaction was studied by recording the $C-V$ characteristics every $2 \mathrm{~min}$ for $10 \mathrm{~min}$. Finally, the measurements were taken at 15, 20, 25 and $30 \mathrm{~min}$ after the start of the phosphorylation reaction. In the control reactions, $C-V$ measurements were taken at similar time intervals. Each experiment was repeated at least three times and the reported data correspond to the average values. The maximum observed value of the capacitance, $C_{\max }$, which corresponds to $C_{\text {dielectric }}$ (capacitance of the silicon nitride/silicon dioxide dielectric layers) does not vary more than $3 \%$ from curve to curve. Therefore, the curves were normalised to $C_{\max }$ plot voltage differences for ease of comparison.

E. LSPR measurements: The instruments for LSPR namely, 14 reflection probe (R400-7UV-VIS), halogen light source (LS-1-LL) and the spectroscope (USB4000-UV-VIS-ES) were purchased from Ocean Optics. Before taking any signal from the scope, the system was calibrated for dark and light spectrum modes. The LSPR signal was then recorded in absorption mode by observing the wavelength dependence of the light absorbed through by nanoparticles via the SpectraSuite software (cross-platform spectroscopy operating software from Ocean Optics). These setups have been routinely used to measure acquire LSPR signals in recent works ${ }^{30}$.

\section{Conflicts of interest}

There are no conflicts to declare.

\section{Acknowledgements}

The authors are thankful to Dr V. Pachauri, Prof S. Ingebrandt (University of Applied Sciences Kaiserslautern) and $\operatorname{Dr} \mathrm{S}$. Sivaraya (University of Bath), Dr Z. Yang (University of Glasgow) for providing silicon nitride wafers, as well as Dr R. Dondi and Dr I. Eggleston for the peptide synthesis.

\section{References}

M. Martin, A.M. Steiner, F. Röder, P. Formanek, T. A.F. König, and Andreas Fery. Angew. Chem. Int. Ed., 2017,50, 15866-15870 Z.Y. Bao, D. Y. Lei, R. Jiang, X. Liu, J. Dai, J. Wang, H. L. W. Chan and Y. H. Tsang, Nanoscale, 2014, 6, 9063-9070

3 Z.Y. Bao, J. Dai, D.Y. Lei, and Y. Wu, J. Appl. Phys., 2013,114, 124305.

4 R. Jiang, B. Li, C. Fang and J. Wang, Adv. Mater., 2014, 26, 5274-5309.

5 J. Y. Oh, H.-J. Jang, W.-J. Cho and M. S. Islam, Sensors Actuators B Chem., 2012, 171-172, 238-243.

6 T. M. Pan, M. De Huang, W. Y. Lin and M. H. Wu, Anal. Chim. Acta, 2010, 669, 68-74.
K. Saha, S. S. Agasti, C. Kim, X. Li and V. M. Rotello, Chem. Rev., 2012, 112, 2739-2779.

M. Chamanzar, Z. Xia, S. Yegnanarayanan and A. Adibi, Opt. Express, 2013, 21, 32086-32098.

P. R. Sajanlal and T. Pradeep, Nanoscale, 2012, 4, 34273437.

P. Vasa, R. Pomraenke, S. Schwieger, Y. I. Mazur, V. Kunets, P. Srinivasan, E. Johnson, J. E. Kihm, D. S. Kim, E. Runge, G. Salamo and C. Lienau, Phys. Rev. Lett., 2008, 101,116801,14

S. Schietinger, M. Barth, T. Aichele and O. Benson, Nano Lett., 2009, 9, 1694-1698.

R. Verre, Z. J. Yang, T. Shegai and M. Käll, Nano Lett., 2015, 15, 1952-1958.

K. Maity, D. K. Panda, E. Lochner and S. Saha, J. Am. Chem. Soc., 2015, 137, 2812-2815.

N. Bhalla, M. Di Lorenzo, G. Pula and P. Estrela, Sci. Rep., 2015, 5, 8687, 1-8.

L. Cui, Y. Li, M. Lu, B. Tang and C. yang Zhang, Biosens. Bioelectron., 2018, 99, 1-7.

T. Endo, S. Yamamura, K. Kerman and E. Tamiya, Anal. Chim. Acta, 2008, 614, 182-189.

A. M. Lipchik, R. L. Killins, R. L. Geahlen and L. L. Parker, Biochemistry, 2012, 51, 7515-7524.

T. Anastassiadis, S. W. Deacon, K. Devarajan, H. Ma and J. R. Peterson, Nat. Biotechnol., 2011, 29, 1039-1045.

F. Wang, D. Li, L. Jin, C. Ren, D. Yang and D. Que, Opt. Lett., 2013, 38, 28321-28324.

T. Yoshinobu, H. Iwasaki, Y. Ui, K. Furuichi, Y. Ermolenko, Y. Mourzina, T. Wagner, N. Näther and M. J. Schöning, Methods, 2005, 37, 94-102.

F. Wang, D. Li, D. Yang and D. Que, Nanoscale Res. Lett., 2012, 7, 669-674.

J. Kistner, X. Chen, Y. Weng, H. P. Strunk, M. B. Schubert and J. H. Werner, J. Appl. Phys., 2011, 110, 23520.

F. Wang, D. Li, D. Yang and D. Que, Nanoscale Res. Lett., 2012, 7, 1-6.

G. Sun, J. B. Khurgin and R. A. Soref, Appl. Phys. Lett., 2009, 94, 101103.

J. Zhang, P. Yang and N. Gray, Nat. Rev. Cancer, 2009, 9, 2839.

C. Holmes and J. Amin, Medicine (Baltimore)., 2016, 44, 687-690.

Y. Deng, B. Li, F. Liu, K. Iqbal, I. Grundke-Iqbal, R. Brandt and C.-X. Gong, FASEB J., 2007, 22, 138-145.

N. Bhalla, M. Di Lorenzo, G. Pula and P. Estrela Biosens. Bioelectron., 2014, 54, 109-114.

N. Formisano, N. Bhalla, L. C. C. Wong, M. Di Lorenzo, G. Pula and P. Estrela, Electrochem. commun., 2015, 57,70-73.

N. Bhalla, D. Lee, S. Sathish, A.Q. Shen, Nanoscale, 2017,9, 547-554 